\title{
A redox-dependent interaction between two electron-transfer partners involved in photosynthesis
}

\author{
Renaud Morales $^{1}$, Marie-Hélène Charon ${ }^{1,+}$, Galina Kachalova ${ }^{1, \$}$, Laurence Serre ${ }^{1, \S}$, \\ Milagros Medina ${ }^{2}$, Carlos Gómez-Moreno ${ }^{2}$ and Michel Frey ${ }^{1,+}$
}

${ }^{1}$ LCCP, Institut de Biologie Structurale J.P. Ebel, CEA-CNRS, 41 rue Jules Horowitz, F38027 Grenoble, France and ${ }^{2}$ Departamento de Bioquímica y Biología Molecular y Celular, Universidad de Zaragoza, 50009-Zaragoza, Spain

Received May 31, 2000; revised July 20, 2000; accepted July 25, 2000

Ferredoxin:NADP ${ }^{+}$:reductase (FNR) catalyzes one terminal step of the conversion of light energy into chemical energy during photosynthesis. FNR uses two high energy electrons photoproduced by photosystem I (PSI) and conveyed, one by one, by a ferredoxin (Fd), to reduce $\mathrm{NADP}^{+}$to NADPH. The reducing power of NADPH is finally involved in carbon assimilation. The interaction between oxidized FNR and Fd was studied by crystallography at $2.4 \AA$ resolution leading to a three-dimensional picture of an Fd-FNR biologically relevant complex. This complex suggests that FNR and Fd specifically interact prior to each electron transfer and disassemble upon a redox-linked conformational change of the $\mathrm{Fd}$.

\section{INTRODUCTION}

Ferredoxin:NADP+reductase (FNR), a flavine-adeninedinucleotide (FAD) flavoenzyme, uses high energy electrons photoproduced by photosystem I (PSI) and protons to reduce nicotinamide-adenine-dinucleotide phosphate $\left(\mathrm{NADP}^{+}\right)$to $\mathrm{NADPH}$ following the reaction $2 \mathrm{Fd}_{\text {red }}+\mathrm{NADP}^{+}+2 \mathrm{H}^{+}<=>2 \mathrm{Fd}_{\text {ox }}$ $+\mathrm{NADPH}+\mathrm{H}^{+}$. The reducing power of NADPH is finally involved in carbon assimilation (Carrillo and Vallejos, 1987; Arakaki et al., 1997). The two electrons involved in NADP+ reduction are transferred, one at a time, from $\mathrm{PSI}$ to $\mathrm{NADP}^{+}$by a [2Fe-2S] ferredoxin ( $\mathrm{Fd})$. Each electron transfer requires the formation of a transient complex between a reduced $\mathrm{Fd}\left(\mathrm{Fd}_{\text {red }}\right)$ and a preformed FNR-NADP ${ }^{+}$complex (Batie and Kamin, 1984b; Sancho and Gómez-Moreno, 1991). More recently, it has been proposed that a larger transient complex involving PSI, Fd and FNR could be formed during linear electron transport (van Thor et al., 1999). We have previously determined the structures of the uncomplexed oxidized (Serre et al., 1996) and reduced ( $R$. Morales, unpublished data) FNR and the oxidized and reduced Fd (Morales et al., 1999) from the cyanobacterium Anabaena PCC7119. To gain further structural insights on this important step of photosynthesis, we have studied by crystallography the interaction between oxidized FNR and Fd, both extracted from the same organism.

\section{RESULTS AND DISCUSSION}

\section{Overall structure}

The two proteins co-crystallized only with a molar excess of FNR over Fd. This results in a crystallographic asymmetric unit that consists of two reductases, named FNR1 and FNR2, and one Fd molecule. FNR1 and FNR2 interact with each other and 'grasp' the globular Fd molecule like a pincer (Figure 1).

\section{Biological relevance}

In the FNR1-Fd-FNR2 crystallographic moiety, the Fd [2Fe-2S] cluster is $7.4 \AA$ distant from the exposed C8-isoalloxazine methyl (C8M) of FNR1 (Figure 1) thought to be involved in electron transfer in flavoproteins (Fritz et al., 1973). Such a close distance between the two redox centers might account for the fast electron

+Corresponding authors. E-mail: frey@lccp.ibs.fr and charon@lccp.ibs.fr

Present address: Max-Planck Institut, Notkestrasse 85, 22603 Hamburg, Germany

\$Present address: CBM-CNRS, rue Charles Sadron, 45071 Orléans Cedex, France 


\section{scientific reports}

\section{R. Morales et al.}

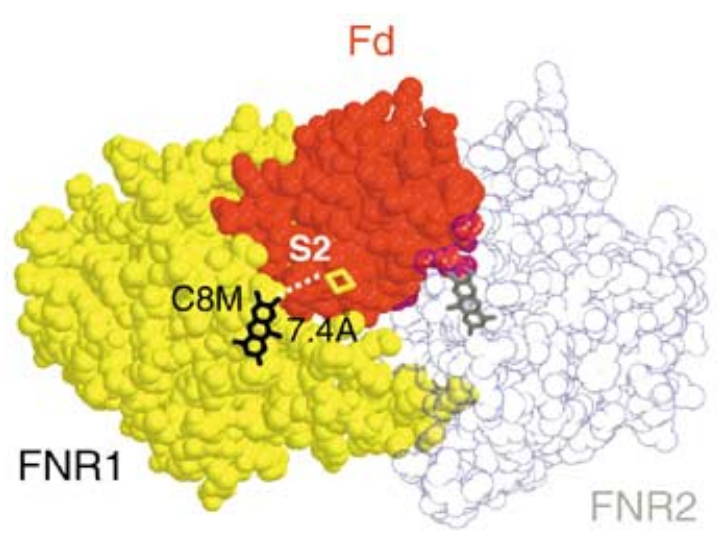

Fig. 1. The crystal asymmetric unit in $\mathrm{CPK}$ mode. The ferredoxin (Fd, in red) is grasped by the two FNR molecules (FNR1 in yellow and FNR2 in light gray). The FAD isoalloxazines and the $[2 \mathrm{Fe}-2 \mathrm{~S}]$ cluster lozenge are represented in black and yellow. The closest distances from the $[2 \mathrm{Fe}-2 \mathrm{~S}]$ cluster Fd to FNR1 and FNR2 isoalloxazine rings are 7.4 and $14.5 \AA$, respectively.

transfer between Anabaena Fd and FNR in solution $\left(6000 \mathrm{~s}^{-1}\right.$; Walker et al., 1991). On the whole, the interactions between $\mathrm{Fd}$ and FNR1 are far more specific than those between Fd and FNR2 in terms, for example, of the number of hydrogen bonds and ion pairs (10 versus 3 ) (Table I), van der Waals contacts [VDW $(<4.1$ $\AA)=54$ versus 21] and buried area upon complex formation $\left(1600 \mathrm{~A}^{2}\right.$ versus $1100 \mathrm{~A}^{2}$ ). These characteristics of interactions are quite comparable to those observed in other crystallographic complexes (for review see Lo Conte et al., 1999). Moreover, the molecular dipoles of Fd and FNR1, as calculated by GRASP (Nicholls et al., 1991), are nearly colinear and orientated in the same direction, resulting in the negative pole of Fd being close to the positive pole of FNR1. The biological relevance of the association between Fd and FNR1 (Fd-FNR1) is also supported by a wealth of studies in solution, mostly on spinach leaves and Anabaena: chemical cross-linking (Zanetti et al., 1988), differential chemical modifications (De Pascalis et al., 1993), limited proteolysis (Gadda et al., 1990) and joint protein engineering and spectroscopy (e.g. Aliverti et al., 1997; Hurley et al., 1997; Martínez-Júlvez et al., 1998, 1999 and citations therein). Nearly all the interactions identified in solution by these various approaches are found at the Fd-FNR1 interface. This strongly

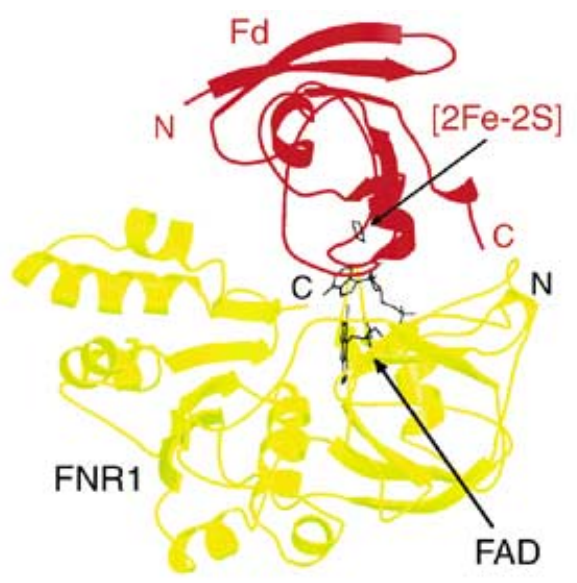

Fig. 2. Fd-FNR1 ribbon diagram of the three-dimensional structure. FNR1 is colored in yellow and ferredoxin in red. The cofactors are represented by thin dark lines and the $\mathrm{C}$ - and $\mathrm{N}$-termini are indicated.

suggests that the binding of a second reductase (FNR2) to FdFNR1 within the crystal does not perturb the association between Fd and FNR.

Moreover, the association of Fd and FNR2 could not represent a catalytically efficient electron transfer complex because the distance between the Fd cluster and the FNR2-FAD is too long for a fast electron transfer (14.5 ̊). The crystallographic moiety FNR1-Fd-FNR2 cannot mimic a complex in vivo mainly because the interactions between the reductases FNR1 and FNR2 interfere with their respective $\mathrm{NADP}^{+}$binding sites (Karplus et al., 1991; Serre et al., 1996). However, the FNR1FNR2 interaction probably has no effect on the Fd-FNR association since $\mathrm{NADP}^{+}$and $\mathrm{Fd}$ bind to distinct sites on FNR, whereas they act in a pattern of negative cooperativity (Batie and Kamin, 1984a). Moreover, such a heterotrimeric moiety would also impair the formation of the transient productive complex PSIFd-FNR, which has recently been described (van Thor et al., 1999). Finally, the role of FNR2 could be to stabilize crystal packing by contacts with neighboring proteins.

\section{Intermolecular interactions}

In Fd-FNR1 (Figure 2), Fd binds to the N-terminal FAD binding domain (residues 12-137) and the C-terminus structural subdomain (residues 237-302) on the concave side of the FNR1

Table I. Fd-FNR1 association: selected ${ }^{\mathrm{a}}$ intermolecular hydrogen bonds

\begin{tabular}{|c|c|c|c|c|c|}
\hline Ferredoxin residues & FNR1 residues & Distances $(\AA)$ D...A & Ferredoxin residues & FNR1 residues & Distances $(\AA)$ D...A \\
\hline Tyr25-On & 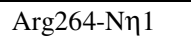 & 2.6 & Asp62-O 82 & Thr302-O $\gamma 1$ & 3.4 \\
\hline Arg 42-O & Arg264-Nך2 & 3.2 & Ser64-O $\gamma$ & Glu301-Oع2 & 2.9 \\
\hline Ser61-O $\gamma$ & Lys293-N & 3.1 & Ser64-O & Val136-O & 3.3 \\
\hline Ser61-O & Val300-O & 3.4 & Asp67-O $\delta 1$ & Asn13-O $\delta 1$ & 3.1 \\
\hline Asp62-O $\delta 1$ & Glu267-Oع 1 & 3.3 & Asp67-O 82 & Asn13-O $\delta 1$ & 2.3 \\
\hline- & Glu267-Oع2 & 3.2 & Glu94-Oع2 & Lys75-N $\zeta$ & 3.0 \\
\hline
\end{tabular}

${ }^{\text {a}}$ Hydrogen bonds $<3.4 \AA$; D-H...A $>90^{\circ}$. 


\section{scientific reports}

Redox-dependent ferredoxin-FNR interaction

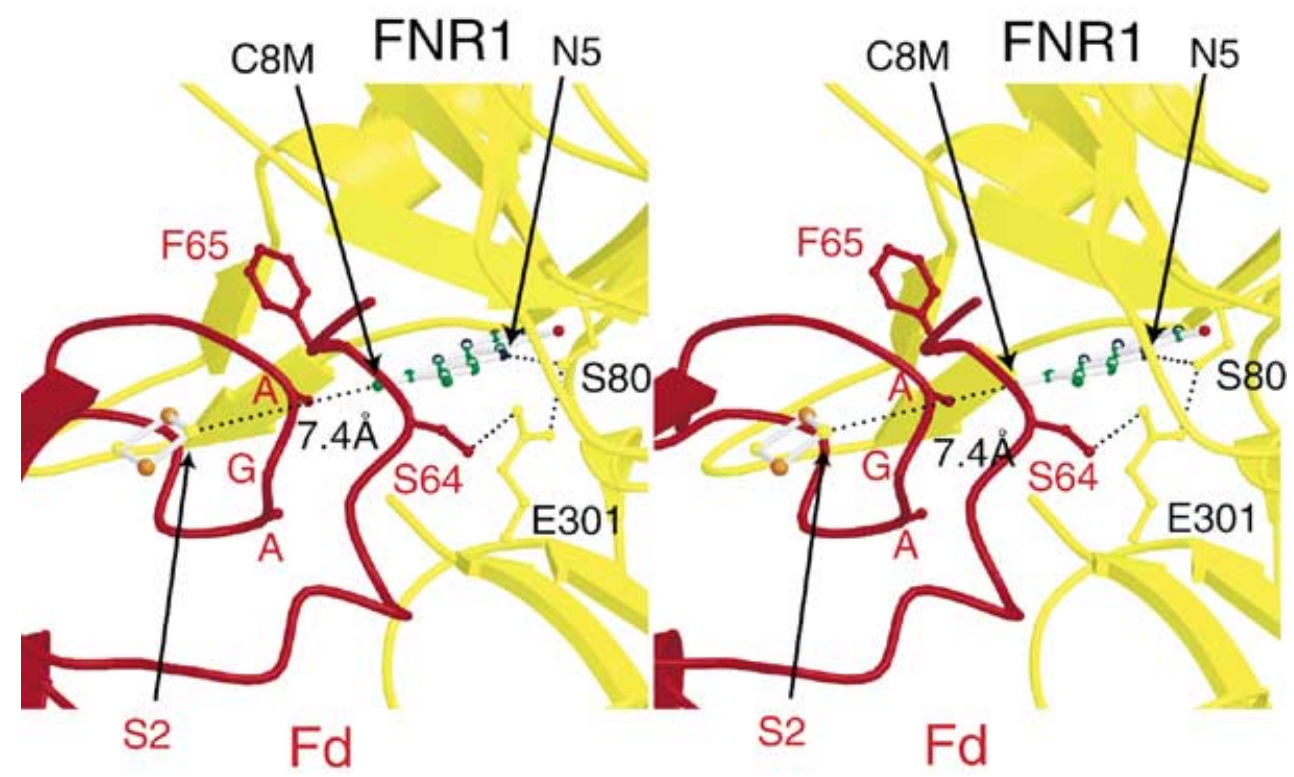

Fig. 3. Fd-FNR1 stereoview showing the arrangement of the two redox centers. Residues in close contact between the [2Fe-2S] cluster and FAD as well as residues possibly involved in proton transfer are represented by balls and sticks.

molecule, which contains the solvent-exposed di-methyl edge of the FAD isoalloxazine. These two domains do not include the $\mathrm{NADP}^{+}$binding site already known (Karplus et al., 1991; Serre et al., 1996). By comparison with the uncomplexed oxidized molecules (Serre et al., 1996; Morales et al., 1999), Fd undergoes a conformational change in binding to FNR1 at the level of the loop 60-67 [the root mean square difference (r.m.s.d.) between the $\mathrm{C}_{\alpha}$ carbons of residues $60-67$ is $2.5 \AA$ ]. This change in particular could be explained by the strong interaction of the $\mathrm{Fd}$ Phe65 aromatic side chains with FNR1 (11 VDW contacts). By contrast, the FNR structural changes upon complexation concern only side chains interacting with surface side chains of the $\mathrm{Fd}$ (Table I). To sum up, the molecular interface between Fd and FNR1 consists of a core of hydrophobic side chains including Fd Phe65 and FNR1 Leu76, Leu78 and Val136, negatively charged groups of the $\mathrm{Fd}$ and positively and some negatively charged groups of FNR. These groups interact with each other either directly or through 'bridging' water molecules. In addition, the Fd-FNR1 interface is stabilized by hydrophobic forces either involving long carbonyl side chains like FNR Arg264 (11 VDW with $\mathrm{Fd}$ ) or resulting from the loss of $\sim 10$ water molecules upon complex formation. The latter point is in accordance with calorimetry experiments on the spinach complex (Jelesarov and Bosshard, 1994).

\section{Comparison with hypothetical Fd-FNR models}

Some hypothetical models of Fd-FNR complexes have been proposed (De Pascalis et al., 1993; Karplus and Bruns, 1994; Fukuyama et al., 1995). Analysis of one of these models (Fukuyama et al., 1995) compared with Fd-FNR1 shows that the core of the interface also consists of an intermolecular patch of the hydrophobic side chains, Fd Phe65 and FNR Leu76, Leu78 and Val136. However, the orientation of the Fd towards FNR, in both the crystallographic and the hypothetical complex, differs by $\sim 30^{\circ}$. This is due to the involvement of either the $\mathrm{Fd}$ acidic patch Asp67-Asp68 in our crystallographic model, or Asp26Glu29-Glu30 (Anabaena numbering) in all the hypothetical models, in the molecular interaction. As shown by differential chemical modification studies on spinach (De Pascalis et al., 1993) both interactions are possible although the protection of Asp67-Asp68 is stronger than that of Asp26-Glu29-Glu30 upon complexation.

\section{Electron and proton transfer}

The arrangement of the redox centers of Fd and FNR1 (Figure 3) is similar to that of the flavin mononucleotide and [2Fe-2S] cluster observed in phthalate dioxygenase reductase (PDR), an NADH oxidizing enzyme that contains one FNR-like and one Fd-like domain in a single polypeptide chain (Correll et al., 1992). However, in PDR, the iron-sulfur cluster atom that is the closest to the FAD C8M is Fe1 (7.9 $\AA$ ) instead of S2 (7.4 $\AA$ ) in FdFNR1. Density functional theory calculations (Noodleman et al., 1995), together with the X-ray structures of the uncomplexed oxidized and reduced $\mathrm{Fd}$, have strongly suggested that upon reduction the change in net charge on Fe1 spreads partially over the S2 sulfur atom of the [2Fe-2S] cluster (Morales et al., 1999). Therefore, the close proximity of S2 to C8M in a dissociable FdFNR complex might aim at a more efficient electron transfer. The respective redox centers of Fd and FNR1 are only separated by an Fd main chain segment of limited steric hindrance, Ala43Gly44-Ala45 (Figure 3), which should allow a 'direct' electron transfer between them. However, our model suggests that, as previously proposed (Hurley et al., 1993), the $\pi$ orbitals of the 


\section{scientific reports}

\section{R. Morales et al.}

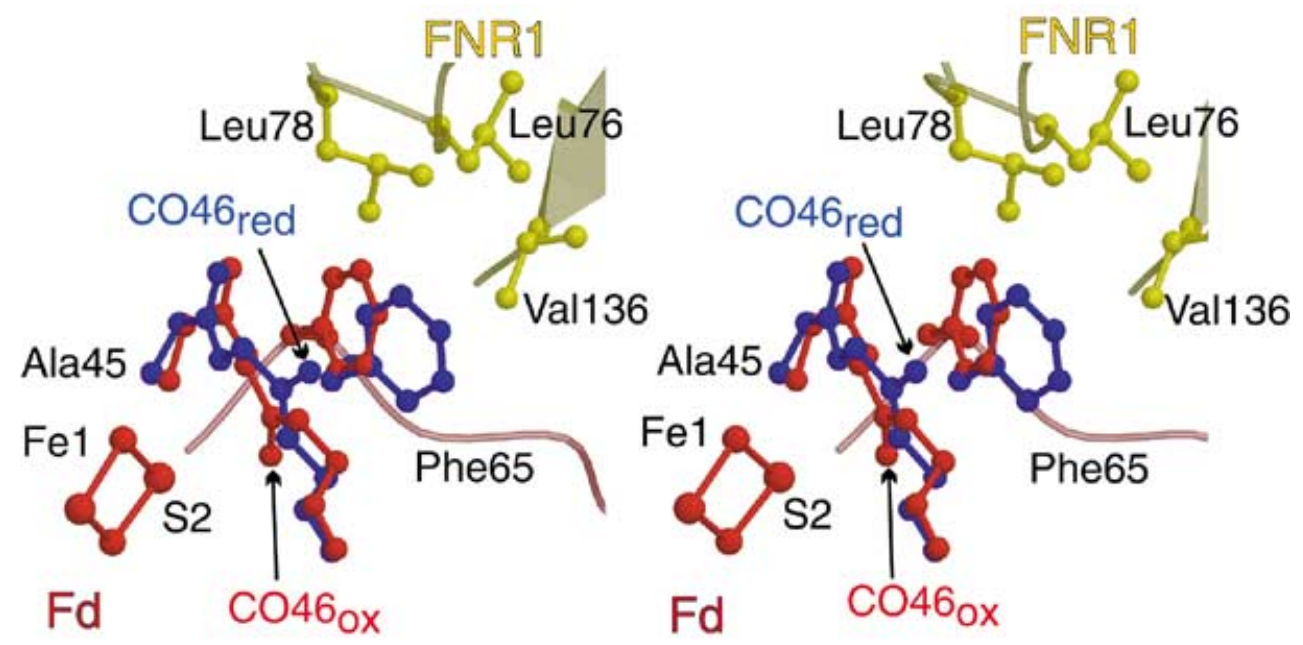

Fig. 4. Stereoview of the reduced Fd crystal structure (in blue) superimposed on Fd-FNR1 (oxidized Fd in red and FNR1 in yellow). Note the redox-linked flip of the peptide bond 46-47 of Fd (Morales et al., 1999) and the intermolecular hydrophobic patch formed by the side chains of Fd Phe65 and FNR Leu76, Leu78 and Val136.

Phe65 aromatic ring could also be involved in the electronic coupling between the two cofactors.

It has been proposed that proton transfer from the external medium to the FNR isoalloxazine N5 atom could be mediated by the solvent-exposed Glu301 and Ser80 in Anabaena FNR (Serre et al., 1996), similar to Glu312 and Ser96 in spinach FNR (Bruns and Karplus, 1995). This mechanism has since been debated on the basis of site-specific mutagenesis studies (e.g. Medina et al., 1998). In our Fd-FNR1 crystallographic association, the carboxylic group of Glu301 is no more exposed to solvent but is hydrogen-bonded to the hydroxyl group of $\mathrm{Fd}$ Ser64 (Ser64-Or), which is in turn exposed to solvent (Figure 3). This is, maybe, not coincidental and puts forward again a possible proton transfer pathway between the external medium and the FNR isoalloxazine N5 through Fd Ser64 and FNR Glu301 and Ser80 side chains.

\section{Catalytic cycle}

After reduction of Fd by PSI (e.g. Barth et al., 1998; van Thor et al., 1999), the first step of the catalytic cycle leading to the reduction of $\mathrm{NADP}^{+}$to $\mathrm{NADPH}$ requires the binding of this first $\mathrm{Fd}_{\text {red }}$ molecule to a preformed $\mathrm{FNR}_{\mathrm{ox}}-\mathrm{NADP}^{+}$complex (Batie and Kamin, 1984b). The FNR ${ }_{\text {ox }}-\mathrm{NADP}^{+}$crystal structure of a pea FNR mutant $(\mathrm{Y} 308 \mathrm{~W})$ suggests that the FNR's invariant Cterminal tyrosine (Tyr303 in Anabaena) is displaced into the solvent so that the $\mathrm{NADP}^{+}$nicotinamide could stack on to the FAD isoalloxazine of FNR (Deng et al., 1999). Molecular modeling shows that the 'displaced' C-terminal Tyr303 might be accommodated either by a cavity between Fd and FNR1 or by the solvent itself without changing our Fd-FNR1 crystallographic complex in accordance with biochemical evidence (Batie and Kamin, 1984b; Sancho and Gómez-Moreno, 1991).

Upon electron transfer to $\mathrm{FNR}$, the resulting $\mathrm{Fd}_{\mathrm{ox}}$ dissociates from the $\mathrm{FNR}_{\text {semiquinone(sq) }}-\mathrm{NADP}^{+}$complex (Batie and Kamin, 1984b). How can this event be triggered? On the one hand, it has been observed, by crystallography, that the peptide bond linking Cys46 and Ser47 points its carbonyl oxygen away from the nearby cluster S2 sulfur atom in the reduced Fd and toward it in the oxidized Fd (Morales et al., 1999) (Figure 4). In any redox state the molecular surface is nearly the same and the 4647 peptide link lies in close contact with the Fd Phe65 aromatic ring. On the other hand, as shown in this paper, Fd Phe65 interacts directly with FNR1. Taken together, these observations strongly suggest that the dissociation of the $\mathrm{FNR}_{\mathrm{sq}}-\mathrm{Fd}_{\mathrm{ox}}$ complex could be triggered by the redox-linked 46-47 peptide 'flip' of the $\mathrm{Fd}$, which transiently moves the aromatic side chain of $\mathrm{Fd}$ Phe65. It is also of interest to note that such a redox-dependent mechanism has been proposed for the dissociation of the oxidized plastocyanin and PSI (Bendall, 1996).

The second reduced $\mathrm{Fd}$ molecule then probably binds to $\mathrm{FNR}_{\mathrm{sq}}-\mathrm{NADP}^{+}$similarly to $\mathrm{FNR}_{\mathrm{ox}}-\mathrm{NADP}^{+}$as we did not observe significant conformational differences between the crystal structures of the oxidized and reduced FNRs (Serre et al., 1996; R. Morales, unpublished data).

\section{Conclusion}

The specificity of the molecular interactions and the molecular dipole complementarity suggest that our Fd-FNR1 crystallographic model represents a highly probable conformational state of an electron transfer complex between Fd and FNR during the last step of photosynthesis. Nevertheless, the hydrophobic patch centered on FNR Leu76, Leu78 and Val136 is sufficiently extended to accommodate other hydrophobic interactions with Fd so that the two redox centers are still close enough to each other for fast electronic exchanges. This should allow a relatively flexible mode of docking and favor turnover, in accordance with kinetics and theoretical studies (Walker et al., 1991). Moreover, our model of a redox-sensitive association between Fd and FNR could be envisaged between Fd and other functional partners such as PSI (e.g. Barth et al., 1998; van Thor et al., 1999), sulfite 


\section{scientific reports}

Redox-dependent ferredoxin-FNR interaction

Table II. X-ray data collection and refinement statistics

\begin{tabular}{|c|c|c|c|}
\hline \multicolumn{2}{|l|}{ Data collection statistics } & \multicolumn{2}{|l|}{ Refinement statistics } \\
\hline Wavelength (̊) & 0.98 & Resolution range $(\AA)$ & $15.0-2.38$ \\
\hline Resolution range $(\AA)$ & $50-2.38$ & $R_{\text {cryst }} / R_{\text {free }}(\%)$ & $21.9 / 29.2$ \\
\hline Unique reflections & 25838 & No. of atoms & 5633 \\
\hline$R_{\mathrm{sym}}(\%)^{\mathrm{a}}$ & $4.6(7.7)^{\mathrm{b}}$ & No. of water molecules & 91 \\
\hline Average $I / \sigma(I)$ & $7.9(7.3)^{\mathrm{b}}$ & Average $B$ value $\left(\AA^{2}\right)$ & 50.1 \\
\hline Redundancy & $4.3(3.7)^{\mathrm{b}}$ & R.m.s. bond distances $(\AA)$ & 0.02 \\
\hline Completeness $(\%)$ & $97.2(87.7)^{\mathrm{b}}$ & R.m.s. angle distances $(\AA)$ & 0.02 \\
\hline
\end{tabular}

${ }^{\mathrm{a}} R_{\text {sym }}=\sum \mid I_{\mathrm{i}}-\langle I>| / \sum\langle I\rangle$, where the summation is over all symmetry-equivalent reflections.

bStatistics for outer shell of 2.38-2.4 $\AA$ data are shown in parentheses.

reductase (Akashi et al., 1999) or thioredoxin reductase (Dai et al., 2000).

\section{METHODS}

Crystallization. Oxidized FNR and Fd were co-crystallized at $20^{\circ} \mathrm{C}$ under conditions similar to those used to grow FNR crystals (Serre et al., 1996), but only in the presence of an excess of FNR over Fd $(1.4 / 1$ or $2 / 1, \mathrm{~mol} / \mathrm{mol})$. It is of interest to note that the use of this 'non-stoichiometric' approach has also been crucial to obtain crystals of several intermolecular complexes such as cytochrome $c$ peroxidase-cytochrome $c$ (Pelletier and Kraut, 1992). The orange-red color of our crystals gave the first indication that they contained FNR and Fd since the crystals of oxidized FNR and Fd are bright yellow and dark red, respectively (Morales et al., 2000).

$\mathrm{X}$-ray data collection, processing and structure determination. $X$-ray diffraction data of a single crystal were collected at $100 \mathrm{~K}$ at the ID2 beamline of the European Synchrotron Radiation Facility (ESRF) (Table II). The crystal cell parameters are: $a=$ $63.73, b=63.72, c=158.02 \AA$ and the space group is $P 2{ }_{1} 2_{1} 2_{1}$, with two FNR and one Fd molecules in the asymmetric unit. The two FNR molecules were positioned by molecular replacement with AMoRe (Navaza, 1994) (correlation coefficient $=0.75$ and $R_{\text {cryst }}=0.30$ ) with the Anabaena FNR (Serre et al., 1996) as a model. The Fd [2Fe-2S] cluster iron atoms were located using the anomalous signal and the molecule itself finally positioned by $R_{\min }$ search techniques. At this stage, crystal packing analysis showed that the crystal could be described as a twin. Interleaving cycles of electron density map calculations, model building and refinement were finally carried out with $\mathrm{O}$ (Jones et al., 1991) and the SHELX97 'twin option' (Sheldrick et al., 1993). The final electron density map accounts for the FNR and Fd amino acid sequences. The final $R$ and $R_{\text {free }}$ factors were 0.22 and 0.29 , respectively (Table II) (Morales et al., 2000).

The figures were prepared with MOLSCRIPT (Kraulis, 1991) and RASTER3D (Merritt and Bacon, 1997).

The atomic coordinates of the complex (1EWY) will be released at the Protein Data Bank upon publication.

\section{ACKNOWLEDGEMENTS}

We thank Dr Bjarne Rasmussen and Xavier Vernède for their help in collecting Synchrotron data at ESRF, Professor Keiichi
Fukuyama for the coordinates of his theoretical model and Professor Hans Bosshard, Dr Jean-Marie Mouesca and Professor Gordon Tollin for helpful suggestions.

\section{REFERENCES}

Akashi, T., Matsumura, T., Ideguchi, T., Iwakiri, K., Kawakatsu, T., Taniguchi, I. and Hase, T. (1999) Comparison of the electrostatic binding sites on the surface of ferredoxin for two ferredoxin-dependent enzymes, ferredoxin-NADP ${ }^{+}$reductase and sulfite reductase. J. Biol. Chem., 274, 29399-29405.

Aliverti, A., Livraghi, A., Piubelli, L. and Zanetti, G. (1997) On the role of the acidic cluster Glu 92-94 of spinach ferredoxin I. Biochim. Biophys. Acta, 1342, 45-50.

Arakaki, A.K., Ceccarelli, E.A. and Carrillo, N. (1997) Plant-type ferredoxin$\mathrm{NADP}^{+}$reductases: a basal structural frame work and a multiplicity of functions. FASEB J., 11, 133-140.

Barth, P., Lagoutte, B. and Sétif, P. (1998) Ferredoxin reduction by photosystem I from Synechocystis sp. PCC 6803: toward an understanding of the respective roles of subunits $\mathrm{PsaD}$ and PsaE in ferredoxin binding. Biochemistry, 37, 16233-16241.

Batie, C.J. and Kamin, H. (1984a) Ferredoxin:NADP ${ }^{+}$oxidoreductase. Equilibria in binary and ternary complexes with $\mathrm{NADP}^{+}$and ferredoxin. J. Biol. Chem., 259, 8832-8839.

Batie, C.J. and Kamin, H. (1984b) Electron transfer by ferredoxin:NADP ${ }^{+}$ reductase. Rapid-reaction evidence for participation of a ternary complex. J. Biol. Chem., 259, 11976-11985.

Bendall, D.S. (1996) Interprotein electron transfer. In Bendall, D.S. (ed.), Protein Electron Transfer. BIOS Scientific Publ. Inc., Oxford, UK, pp. 43-68.

Bruns, C. and Karplus, P.A. (1995) Refined crystal structure of spinach ferredoxin reductase at $1.7 \AA$ resolution: oxidized, reduced and 2'-phospho-5'AMP bound states. J. Mol. Biol., 247, 125-145.

Carrillo, N. and Vallejos, R.H. (1987) Ferredoxin-NADP ${ }^{+}$oxidoreductase. In Barber, J. (ed.), Topics in Photosynthesis. Vol. 8. Elsevier, Amsterdam, The Netherlands, pp. 527-560.

Correll, C.C., Batie, C.J., Ballou, D.P. and Ludwig, M.L. (1992) Phthalate dioxygenase reductase: a modular structure for electron transfer from pyridine nucleotides to [2Fe-2S]. Science, 258, 1604-1610.

Dai, S., Schwendtmayer, C., Schurmann, P., Ramaswamy, S. and Eklund, H. (2000) Redox signaling in chloroplasts: cleavage of disulfides by an ironsulfur cluster. Science, 287, 655-658.

Deng, Z. et al. (1999) A productive NADP ${ }^{+}$binding mode of ferredoxin$\mathrm{NADP}^{+}$reductase revealed by protein engineering and crystallographic studies. Nature Struct. Biol., 6, 847-853.

De Pascalis, A.R., Jelesarov, I., Ackermann, F., Koppenol, W.H., Hirasawa, M., Knaff, D.B. and Bosshard, H.R. (1993) Binding of ferredoxin to ferredoxin:NADP ${ }^{+}$oxidoreductase: the role of carboxyl groups, electrostatic 


\section{scientific reports}

\section{R. Morales et al.}

surface potential, and molecular dipole moment. Protein Sci., 2, 11261135.

Fritz, J., Müller, F. and Mayhew, S.G. (1973) Electron-nuclear double resonance study of flavodoxin from Peptostreptococcus elsdenii. Helv. Chim. Acta, 56, 2250-2254.

Fukuyama, K., Ueki, N., Nakamura, H., Tsukihara, T. and Matsubara, H. (1995) Tertiary structure of [2Fe-2S] ferredoxin from Spirulina platensis refined at $2.5 \AA$ resolution: structural comparisons of plant-type ferredoxins and an electrostatic potential analysis. J. Biochem. (Tokyo), 117, 1017-1023.

Gadda, G., Aliverti, A., Ronchi, S. and Zanetti, G. (1990) Structure-function relationship in spinach ferredoxin-NADP ${ }^{+}$reductase as studied by limited proteolysis. J. Biol. Chem., 265, 11955-11959.

Hurley, J.K., Cheng, H., Xia, B., Markley, J.L., Medina, M., Gomez-Moreno, C. and Tollin, G. (1993) An aromatic amino acid is required at position 65 in Anabaena ferredoxin for rapid electron transfer to ferredoxin:NADP ${ }^{+}$ reductase. J. Am. Chem. Soc., 115, 11698-11701.

Hurley, J.K. et al. (1997) Structure-function relationships in Anabaena ferredoxin: correlations between X-ray crystal structures, reduction potentials, and rate constants of electron transfer to ferredoxin:NADP ${ }^{+}$reductase for site-specific ferredoxin mutants. Biochemistry, 36, 11100-11117.

Jelesarov, I. and Bosshard, H.R. (1994) Thermodynamics of ferredoxin binding to ferredoxin:NADP ${ }^{+}$reductase and the role of water at the complex interface. Biochemistry, 33, 13321-13328.

Jones, T.A., Zou, J.-Y., Cowan, S.W. and Kjeldgaard, M. (1991) Improved methods for building protein models in electron density maps and the location of errors in these models. Acta Crystallogr. A, 47, 110-119.

Karplus, P.A. and Bruns, C.M. (1994) Structure-function relations for ferredoxin reductase. J. Bioenerg. Biomembr., 26, 89-99.

Karplus, P.A., Daniels, M.J. and Herriott, J.R. (1991) Atomic structure of ferredoxin-NADP ${ }^{+}$reductase: prototype for a structurally novel flavoenzyme family. Science, 251, 60-66.

Kraulis, P.J. (1991) MOLSCRIPT: a program to produce both detailed and schematic plots of protein structures. J. Appl. Crystallogr., 24, 946-950.

Lo Conte, L., Chothia, C. and Janin, J. (1999) The atomic structure of protein-protein recognition sites. J. Mol. Biol., 285, 2177-2198.

Martínez-Júlvez, M., Medina, M., Hurley, J.K., Hafezi, R., Brodie, T.B., Tollin, G. and Gómez-Moreno, C. (1998) Lys75 of Anabaena ferredoxin$\mathrm{NADP}^{+}$reductase is a critical residue for binding ferredoxin and flavodoxin during electron transfer. Biochemistry, 37, 13604-13613.

Martínez-Júlvez, M., Nogues, I., Medina, M. and Gómez-Moreno, C. (1999) Molecular recognition between ferredoxin-NADP ${ }^{+}$reductase and its partners: role of charged and hydrophobic residues. In Ghisla, S., Kroneck, R., Macheroux, P. and Sund, H. (eds), Flavins and Flavoprotein. Rudolf Weber, Berlin, Germany, pp. 741-748.

Medina, M., Martínez-Júlvez, M., Hurley, J.K., Tollin, G. and GómezMoreno, C. (1998) Involvement of glutamic acid 301 in the catalytic mechanism of ferredoxin-NADP ${ }^{+}$reductase from Anabaena PCC 7119. Biochemistry, 37, 2715-2728.
Merritt, E.A. and Bacon, D.J. (1997) Raster3D: photorealistic molecular graphics. Methods Enzymol., 277, 505-524.

Morales, R., Charon, M.-H., Hudry-Clergeon, G., Pétillot, Y., Norager, S., Medina, M. and Frey, M. (1999) Refined X-ray structures of the oxidized, at $1.3 \AA$, and reduced, at $1.17 \AA,[2 \mathrm{Fe}-2 \mathrm{~S}]$ ferredoxin from the cyanobacterium Anabaena PCC7119 show redox-linked conformational changes. Biochemistry, 38, 15764-15773.

Morales, R., Kachalova, G., Vellieux, F., Charon, M.H. and Frey, M. (2000) Crystallographic studies of the interaction between the ferredoxin: $\mathrm{NADP}^{+}$:reductase and ferredoxin from the cyanobacterium Anabaena: looking for the elusive ferredoxin molecule. Acta Crystallogr. D, 56, in press.

Navaza, J. (1994) AMoRe: an automated package for molecular replacement. Acta Crystallogr. A, 50, 157-163.

Nicholls, A., Sharp, K.A. and Honig, B. (1991) Protein folding and association: insights from the interfacial and thermodynamic properties of hydrocarbons. Proteins, 11, 281-296.

Noodleman, L., Peng, C.Y., Case, D.A. and Mouesca, J.-M. (1995) Orbital interactions, electron delocalization and spin coupling in iron-sulfur clusters. Coord. Chem. Rev., 144, 199-244.

Pelletier, H. and Kraut, J. (1992) Crystal structure of a complex between electron transfer partners, cytochrome $c$ peroxidase and cytochrome $c$. Science, 258, 1748-1755.

Sancho, J. and Gómez-Moreno, C. (1991) Interaction of ferredoxin-NADP+ reductase from Anabaena with its substrates. Arch. Biochem. Biophys., 288, 231-238.

Serre, L., Vellieux, F.M.D., Medina, M., Gómez-Moreno, C., FontecillaCamps, J.C. and Frey, M. (1996) X-ray structure of the ferredoxin$\mathrm{NADP}^{+}$reductase from the cyanobacterium Anabaena $\mathrm{PCC} 7119$ at $1.8 \AA$ resolution, and crystallographic studies of $\mathrm{NADP}^{+}$binding at $2.25 \AA$ resolution. J. Mol. Biol., 263, 20-39.

Sheldrick, G.M., Dauter, Z., Wilson, K.S., Hope, H. and Sieker, L.C. (1993) The application of direct methods and Patterson interpretation to high resolution native protein data. Acta Crystallogr. D, 49, 18-23.

van Thor, J.J., Geerlings, T.H., Matthijs, H.C. and Hellingwerf, K.J. (1999) Kinetic evidence for the PsaE-dependent transient ternary complex photosystem I/ferredoxin/ferredoxin:NADP ${ }^{+}$reductase in a cyanobacterium. Biochemistry, 38, 12735-12746.

Walker, M.C., Pueyo, J.J., Navarro, J.A., Gómez-Moreno, C. and Tollin, G. (1991) Laser flash photolysis studies of the kinetics of reduction of ferredoxins and ferredoxin-NADP ${ }^{+}$reductases from Anabaena PCC7119 and spinach: electrostatic effects on intracomplex electron transfer. Arch. Biochem. Biophys., 287, 351-358.

Zanetti, G., Morelli, D., Ronchi, S., Negri, A., Aliverti, A. and Curti, B. (1988) Structural studies on the interaction between ferredoxin and ferredoxin-NADP ${ }^{+}$reductase. Biochemistry, 27, 3753-3759.

DOI: $10.1093 /$ embo-reports/kvd057 